\title{
Bortezomib-based treatment of acute antibody-mediated rejection: a case report
}

\author{
Q. Wang ${ }^{1,3}$, X.L. Li ${ }^{2}$, X.G. Xü ${ }^{3}$, B.Y. Shi ${ }^{3}$, Z.M. Zhang ${ }^{3}$, Z.L. Li ${ }^{3}$, Y. Han $^{3}$, \\ W.Q. Zhou ${ }^{3}$, C.Q. Chen ${ }^{3}$, M. Cai ${ }^{3}$ and X. Zhang ${ }^{1}$ \\ 1'Department of Urology, General Hospital of Chinese PLA, Beijing, China \\ ${ }^{2}$ Cadre Ward, the 309th Hospital of Chinese PLA, Beijing, China \\ ${ }^{3}$ Organ Transplant Research Institute, the 309th Hospital of Chinese PLA, Beijing, \\ China \\ Corresponding authors: M. Cai / X. Zhang \\ E-mail: MingCaidoc@163.com / xzhang@foxmail.com \\ Genet. Mol. Res. 14 (4): 17951-17958 (2015) \\ Received August 18, 2015 \\ Accepted October 24, 2015 \\ Published December 22, 2015 \\ DOI http://dx.doi.org/10.4238/2015.December.22.20
}

ABSTRACT. Antibody-mediated rejection (AMR) is an important factor affecting survival after renal transplantation. A highly selective proteasome inhibitor, bortezomib, clears activated plasma cells from the body and has important therapeutic effect on AMR. We investigated the effects of bortezomib on AMR in a patient after a second renal transplant. Biopsy confirmed the diagnosis of mixed cellular rejection and AMR. Bortezomib was administered on day $1\left(1.3 \mathrm{mg} / \mathrm{m}^{2}\right)$, day $4\left(1.0 \mathrm{mg} / \mathrm{m}^{2}\right)$, and day $8(1.0$ $\left.\mathrm{mg} / \mathrm{m}^{2}\right)$. On the same days, $250 \mathrm{mg}$ methylprednisolone was administered once, and cyclosporine dose $\left(5 \mathrm{mg} \cdot \mathrm{kg}^{-1} \cdot \mathrm{day}^{-1}\right)$ was reduced by $50 \%$. Oral mycophenolate mofetil and steroid were withdrawn on day 1 of bortezomib treatment. Intermittent double-filtration plasmapheresis was also performed. We monitored parameters, including T lymphocyte subsets, CD139 and CD19 expression, panel reactive antibody (PRA), and serum creatinine concentration. At follow-up 6 months after bortezomib treatment, we observed: 1) serum creatinine stabilized at $130 \mu \mathrm{M}$ from a peak level of $337 \mu \mathrm{M}$; 2) PRA decreased from a maximum of 66.7 to $0 \%$; 3) blood plasma cell percentage rebounded after significantly decreasing following 
the first dose of bortezomib; 4) in renal allograft biopsy, immunohistochemical staining for $\mathrm{C} 4 \mathrm{~d}$ shifted from strongly positive to negative, and cellular rejection shifted from type IIA to borderline; and 5) adverse effects such as platelet suppression, hypotension, and grade 3 peripheral neuropathy emerged. Bortezomib effectively treated antibody-mediated renal transplantation rejection in this case study, but clinical trials with large sample sizes are still needed to explore clinical safety and tolerability.

Key words: Bortezomib; Antibody-mediated rejection; Renal transplantation

\section{INTRODUCTION}

Antibody-mediated rejection (AMR) is a significant cause of kidney allograft dysfunction and graft loss (Ruangkanchanasetr et al., 2014), and treatment of AMR after renal transplantation greatly affects the short-term and long-term survival of the transplanted kidney (Everly et al., 2009a). Currently, there are several therapies that may be used to prevent and treat AMR, including plasmapheresis, immunoadsorption, polyclonal antibodies, anti-CD20 monoclonal antibodies (Doxiadis and Claas, 2009; Iordan et al., 2009), immune globulin, rituximab, rabbit antithymocyte globulin (rATG), and eculizumab (Burton et al., 2015). However, since these methods do not eliminate or decrease the function of plasma cells, their therapeutic efficacy on AMR is often incomplete or short-lived (Perry et al., 2008, 2009; Stegall et al., 2009).

As a highly selective proteasome inhibitor, bortezomib is currently used to eliminate malignant plasma cells in patients with multiple myeloma (Gomez et al., 2014). Recently, a number of studies have shown that bortezomib can effectively remove anti-human leukocyte antigen (antiHLA) antibodies and treat AMR via depletion of plasma cells (Everly et al., 2008, 2009b; Stegall and Gloor, 2010; Wahrmann et al., 2010), but data on the efficacy and safety of bortezomib for the treatment of AMR are limited (Zinn et al., 2014). One study indicated that bortezomib could be an alternative therapeutic option for desensitization and treatment of acute AMR that is unresponsive to conventional therapies (Yang et al., 2014). Another study, which investigated bortezomib as a novel therapy for late AMR of renal allografts, determined that the benefits of bortezomib-based therapy needed to be weighed against the potential adverse effects, especially in older patients and patients with large antibody loads or chronic allograft vasculopathy (Gupta et al., 2014). In this case study, our center for the first time combined bortezomib and double filtration plasmapheresis (DFPP) to treat one case of AMR after second renal transplantation. Clinical diagnosis and treatment strategies for this case are summarized in this report to provide a clinical reference for the application of bortezomib in renal transplant recipients in China.

\section{MEDICAL HISTORY}

\section{Treatment process}

The female patient was 49 years old and hepatitis C-positive. The first renal transplantation was performed in October 1990, and postoperative immunosuppressive regimen was CsA (Jiangsu Safe Pharmaceutical Co. Ltd., Wuxi, China) + Aza (Heumann Pharma, Nuremberg, Germany) + Pred (NAPP Pharmaceuticals Ltd, Cambridge, UK). In 2000, the blood creatinine (Cr) level of the patient became increasingly elevated (perhaps related to irregular intake of anti-rejection drugs), 
and dialysis was resumed beginning in March 2002. In June 2005, the transplant kidney was removed because of high fever, and a second renal transplantation was performed in May 2010. There was no mismatch at $4 \mathrm{HLA}$ sites; there was mismatch at 2 other loci. Preoperative panel reactive antibody (PRA) type II was 46 , and type I was $0 \%$. Pre-sensitized specific antibodies (DRw52, DR08, and DR04) of the recipient were avoided. Rabbit antithymocyte globulin (rATG) was used for immune induction at $50 \mathrm{mg} /$ day for a total of five doses at $0,1,2,3$, and 4 days post-transplantation. Immunosuppressive regimen was CsA (Jiangsu Safe Pharmaceutical Co. Ltd., Wuxi, China) + MMF (Roche, Basel, Switzerland) + Pred (NAPP Pharmaceuticals Limited, Cambridge, UK). This study was conducted in accordance with the declaration of Helsinki. This study was conducted with approval from the Ethics Committee of the 309th Hospital of Chinese PLA. Written informed consent was obtained from the participant.

Acute rejection occurred 20 days after transplantation, and biopsy confirmed mixed cellular rejection and AMR. Bortezomib (Ben Venue Laboratories Inc., Ohio, USA) was administered on day $1\left(1.3 \mathrm{mg} / \mathrm{m}^{2}\right)$, day $4\left(1.0 \mathrm{mg} / \mathrm{m}^{2}\right)$, and day $8\left(1.0 \mathrm{mg} / \mathrm{m}^{2}\right)$. On days 1,4 , and 8 of bortezomib administration, the patient was administered $250 \mathrm{mg}$ methylprednisolone (Pfizer Manufacturing, New York, USA), the dose of cyclosporine was reduced by $50 \%$, and oral mycophenolate mofetil and steroid were withdrawn. In addition, intermittent DFPP was administrated.

\section{TREATMENT OUTCOME}

\section{Change in blood $\mathrm{Cr}$}

After renal transplantation, blood $\mathrm{Cr}$ level of the recipient, as determined by enzymatic method, recovered to $84.6 \mu \mathrm{M}$ and gradually increased to a maximum of $337 \mu \mathrm{M}$. Through comprehensive treatment, including plasmapheresis and bortezomib, blood $\mathrm{Cr}$ was stabilized at around $130 \mu \mathrm{M}$ six months after transplantation (Figure 1A).
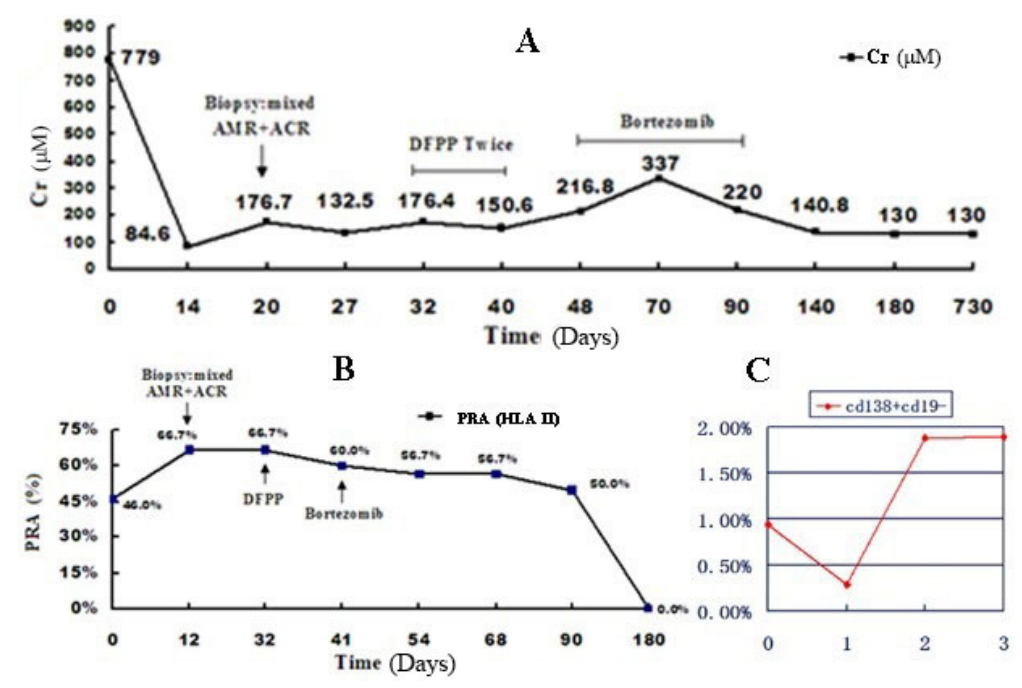

Figure 1. Changes in blood $\mathrm{Cr}$ after renal transplantation (A), in PRA (HLA II) (B), and in CD138+CD19- cells (C) before and after bortezomib treatment AMR antibody-mediated rejection. Days 0, 1, 2 and 3 after bortezomib treatment. 


\section{Pathological changes}

Graft kidney biopsy was performed to perform immunohistochemical staining of the sample, which was evaluated according to the Banff 97 standard classification (2005 update) (Solez et al., 2007). C4d immunohistochemical staining was performed in biopsies from before and after treatment and all evaluations were conducted by the same pathologist (L.A.). The results confirmed the diagnosis of mixed cellular rejection and AMR: C4d staining was strongly positive for AMR, and PRA was positive for acute cellular rejection. After plasmapheresis and bortezomib treatment, repeated transplant biopsy confirmed critical cellular rejection and AMR. As shown in Figure 2, before treatment, surrounding vasculature and interstitium were infiltrated by a large number of lymphocytes, which were significantly reduced after treatment. In addition, both C4d and peritubular capillary (PTC) were positive before treatment and were negative after treatment.

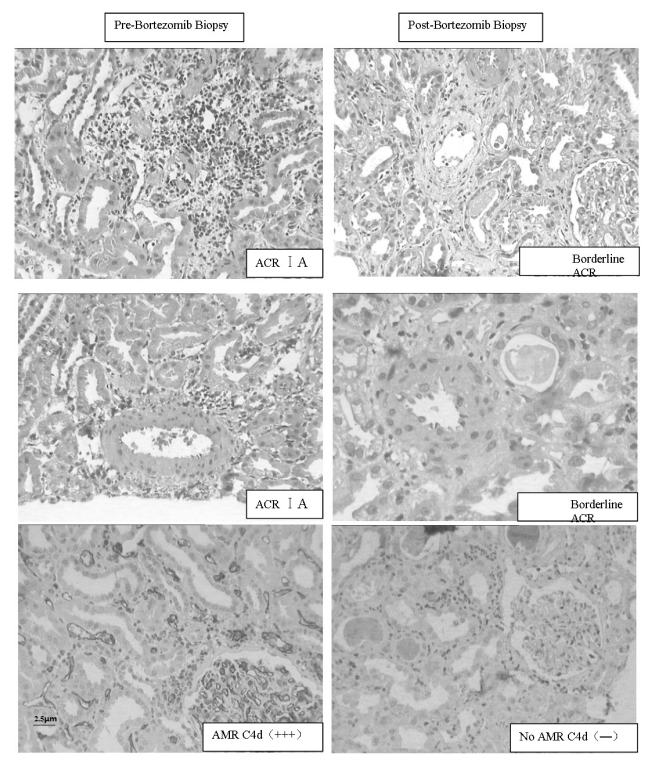

Figure 2. Pathological changes in biopsy before and after renal transplantation.

\section{Changes in PRA}

PRAs were detected by enzyme-linked immunosorbent assay (ELISA). Before transplantation, PRA type II was $46 \%$ and type I was $0 \%$. Specific pre-sensitized antibodies of the recipient (DRw52, DR08 and DR04) were avoided. PRA type II began to increase 2 weeks after the operation to a maximum of $66.7 \%$, while PRA type I remained at $0 \%$. Follow-up after bortezomib treatment (6 months after operation) showed reduction of PRA type II to $0 \%$, with PRA type I also at $0 \%$ (Figure 1B).

\section{Changes in plasma cell expression}

The expression of CD138 and CD19 and plasma cells (CD138+CD19-) before and after bortezomib treatment were detected by flow cytometry, as shown in Figure $1 \mathrm{C}$. There were 
$0.94 \%$ CD138+CD19- cells before treatment, which was reduced to $0.29 \%$ after the first dose of bortezomib. However, the percentage of CD138+CD19- cells rebounded to $1.88 \%$ after the second dose and to $1.89 \%$ after the third dose of bortezomib.

\section{Clinical side effects}

In this case study, the following side effects were positively correlated with bortezomib dose: fatigue, dizziness, low blood pressure, stomach discomfort, frequent nausea with no vomiting, and occasional transient headache. Anuria occurred four hours after treatment, which required dialysis to be performed once and subsided after two days. Symptoms of grade 3 peripheral neuropathy, such as perioral and limb numbness and soreness, indicated impaired nerve function and affected normal daily life. Thrombocytopenia also appeared after treatment but subsided after the drug was withdrawn. Bortezomib also seems to increase the effective concentration of cyclosporine by $\sim 100 \mathrm{mg}$ bid; the peak levels of CsA (C 2) $80 \mu \mathrm{M}$ was used before bortezomib application, which was increased to $1430 \mu \mathrm{M}$ after bortezomib application. Since the patient could not tolerate the significant side effects, the fourth dose of bortezomib was not administered.

\section{LITERATURE REVIEW AND DISCUSSION}

With increasingly successful rates of renal transplantation and the emergence of new efficient immunosuppressants, indications for renal transplantation are also expanding, and more high-risk patients are receiving renal transplantation (Salzedas-Netto et al., 2014). So-called "highly sensitized patients" refer to recipients with factors most likely endangering their lives or shortterm/long-term graft survival, such as second transplantation, multiple transfusions, long-term hemodialysis, and pregnancy, which sensitize the recipient and induce production of a wide range of polyvalent anti-antibodies (Nishio-Lucar et al., 2013). As a result, these recipients are prone to hyperacute transplantation rejections, leading to severe graft failure and even endangerment of their lives (Everly et al., 2009a).

Humoral AMR is one of the major reasons for graft loss in highly sensitized renal transplant patients (Everly et al., 2009). Currently, there are a variety of regimens for the treatment of AMR, such as intravenous immunoglobulin (IVIg), plasmapheresis, rATG, and rituximab. However, efficacy of these treatments has not been confirmed, and the following limitations exist (Perry et al., 2008, 2009; Stegall et al., 2009; Gomez et al., 2014): 1) slow reversal of AMR; 2) cost-ineffectiveness; 3 ) rejection-reversal rates of less than $80 \%$; 4) chronic rejection; and 5 ) persistence of donor-specific anti-HLA antibody after treatment. The above limitations may be due to lack of direct effect on mature plasma cells. As a highly selective proteasome inhibitor, bortezomib can remove mature plasma cells from the body and effectively treat AMR (Doxiadis and Claas, 2009; Perry et al., 2009).

The mechanism of action of bortezomib in the treatment of AMR may be as follows (Vanderlugt et al., 2000; Hideshima et al., 2001; Luo et al., 2001; Adams et al., 2004; Sun et al., 2004; Chauhan et al., 2005; Chromik et al., 2006; Nencioni et al., 2006a,b): 1) to inhibit proteasomes and decrease protein degradation, thereby inhibiting nuclear factor kappa $\mathrm{B}(\mathrm{NF}-\kappa \mathrm{B}$; transcription factor) and partially deregulating the protein synthesis process, leading to accumulation of a large amount of defective ribosome in the endoplasmic reticulum and eventually causing plasma cell apoptosis; 2) to inhibit production of interleukin (IL)-6 by bone marrow stromal cells, resulting in apoptosis of B cells at various differentiation stages (Hideshima et al., 2001); 3) to induce apoptosis 


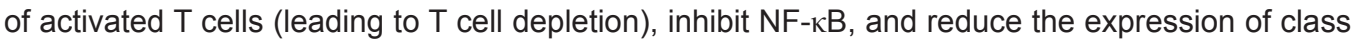
I major histocompatibility complexes and the Th1 response (Vanderlugt et al., 2000; Luo et al., 2001; Adams et al., 2004; Sun et al., 2004; Chauhan et al., 2005; Nencioni et al., 2006a); and 4) to affect dendritic cell function through proteasome inhibition, thereby reducing the expression of costimulatory molecules, decreasing cytokine production, and promoting apoptosis (Chromik et al., 2006; Nencioni et al., 2006a,b).

Some overseas studies (Idical et al., 2008; Perry et al., 2009; Gomez et al., 2014) suggested that one course of bortezomib treatment should include administration of $1.3 \mathrm{mg} /$ $\mathrm{m}^{2}$ bortezomib on days 1, 4, 8, and 11. Generally, use of two or more courses is more effective to clear antibodies. Combination therapy with plasmapheresis, high-dose steroid, or CD20 monoclonal antibody (rituximab) can significantly increase the clinical efficacy of bortezomib (Hosoba et al., 2015).

In this study, we administered bortezomib at doses of $1.3 \mathrm{mg} / \mathrm{m}^{2}$ at day $1,1.0 \mathrm{mg} / \mathrm{m}^{2}$ at day 4 , and $1.0 \mathrm{mg} / \mathrm{m}^{2}$ at day 8 , in combination with methylprednisolone and DFPP adjuvant therapies. Our clinical efficacy was confirmed through clinical and pathological observations, including follow-up at six months post-transplantation. However, due to significant side effects, we were not able to administer bortezomib at the regimen reported in studies conducted overseas. The optimal treatment course of bortezomib for AMR in Chinese patients can only be determined through clinical trials and observations conducted in China.

We observed significant clinical pathological changes after bortezomib treatment, i.e., lymphocyte infiltration, inflammatory changes of capillaries, C4d deposition, and other changes, which have been confirmed by another study (Nowosiad-Magda et al., 2014). The PRA decreased slowly, suggesting that the efficacy of AMR treatment is not only related to the variety of the antibodies in the body but also, more pertinently, to the strength of these antibodies. Overseas studies showed that bortezomib is more effective for AMR when the mean fluorescence intensity (MFI) of the antibody is $>10000$, while an MFI $<10000$ is associated with only partial remission or poor outcome (Gomez et al., 2014).

Overseas studies have also reported common side effects of bortezomib, including inhibition of normal plasma cells, gastrointestinal reactions, thrombocytopenia, and paresthesia (Pai et al., 2014). Our study showed that Chinese patients had poor tolerability for this drug and side effects are obvious, limiting the clinical application of bortezomib in China. Therefore, the dose and frequency of bortezomib treatment in China still needs to be investigated in more clinical trials.

In summary, plasma cell-targeted bortezomib therapy can significantly clear anti-HLA antibodies from the body and potentially address refractory rejection after renal transplantation. Bortezomib is a promising treatment for AMR of renal transplants, but its long-term clinical efficacy and complications need to be explored further.

\section{CONCLUSIONS}

Bortezomib can effectively treat AMR after renal transplantation. However, because of its relatively significant side effects, clinical trials need to be performed on drug safety and tolerability.

\section{Conflicts of interest}

The authors declare no conflict of interest. 


\section{REFERENCES}

Adams J (2004). The proteasome: a suitable antineoplastic target. Nat. Rev. Cancer 4: 349-360.

Burton SA, Amir N, Asbury A, Lange A, et al. (2015). Treatment of Antibody Mediated Rejection in Renal Transplant Patients: A Clinical Practice Survey. Clin. Transplant. 29: 118-123.

Chauhan D, Catley L, Li G, Podar K, et al. (2005). A novel orally active proteasome inhibitor induces apoptosis in multiple myeloma cells with mechanisms distinct from Bortezomib. Cancer Cell. 8: 407-419.

Chromik J, Schnürer E, Georg Meyer R, Wehler T, et al. (2006). Proteasome-inhibited dendritic cells demonstrate improved presentation of exogenous synthetic and natural HLA-class I peptide epitopes. J. Immunol. Methods 308: 77-89.

Doxiadis II and Claas FH (2009). Transplantation of highly sensitized patients via the acceptable mismatch program or desensitization? We need both. Curr. Opin. Organ. Transplant. 14: 410-413

Everly JJ, Walsh RC, Alloway RR and Woodle ES (2009b). Proteasome inhibition for antibody-mediated rejection. Curr. Opin. Organ. Transplant. 14: 662-666.

Everly MJ, Everly JJ, Susskind B, Brailey P, et al. (2008). Bortezomib provides effective therapy for antibody-and cell-mediated acute rejection. Transplantation 86: 1754-1761.

Everly MJ, Everly JJ, Arend LJ, Brailey P, et al. (2009a). Reducing de novo donor-specific antibody levels during acute rejection diminishes renal allograft loss. Am. J. Transplant. 9: 1063-1071.

Gomez AM, Willcox N, Vrolix K, Hummel J, et al. (2014). Proteasome inhibition with bortezomib depletes plasma cells and specific autoantibody production in primary thymic cell cultures from early-onset myasthenia gravis patients. J. Immunol. 193: 1055-1063.

Gupta G, Abu Jawdeh BG, Racusen LC, Bhasin B, et al. (2014). Late antibody-mediated rejection in renal allografts: outcome after conventional and novel therapies. Transplantation 97: 1240-1246.

Hideshima T, Richardson P, Chauhan D, Palombella VJ, et al. (2001). The proteasome inhibitor PS-341 inhibits growth, induces apoptosis, and overcomes drug resistance in human multiple myeloma cells. Cancer Res. 61: 3071-3076.

Hosoba S, Jaye DL, Cohen C, Roback JD, et al. (2015). Successful treatment of severe immune hemolytic anemia after allogeneic stem cell transplantation with bortezomib: report of a case and review of literature. Transfusion 55: 59-264.

Idical A, Kaneku H, Everly MJ, Trivedi HL, et al. (2008). Elimination of post-transplant donor-specific HLA antibodies with bortezomib. Clin. Transpl. 229-239.

lordan SC, Peng A and Vo AA (2009). Therapeutic strategies in management of the highly HLA-sensitized and ABOincompatible transplant recipients. Contrib. Nephrol. 162: 13-26.

Luo H, Wu Y, Qi S, Wan X, et al. (2001). A proteasome inhibitor effectively prevents mouse heart allograft rejection. Transplantation 72: 196-202.

Nencioni A, Garuti A, Schwarzenberg K, Cirmena G, et al. (2006a). Proteasome inhibitor induced apoptosis in human monocyte-derived dendritic cells. Eur. J. Immunol. 36: 681-689.

Nencioni A, Schwarzenberg K, Brauer KM, Schmidt SM, et al. (2006b). Proteasome inhibitor bortezomib modulates TLR4induced dendritic cell activation. Blood 108: 551-558.

Nishio-Lucar A, Balogun RA and Sanoff S (2013). Therapeutic apheresis in kidney transplantation: a review of renal transplant immunobiology and current interventions with apheresis medicine. J. Clin. Apher. 28: 56-63.

Nowosiad-Magda M, Myślak M, Roszkowska P, Borowiec-Chłopek Z, et al. (2014). Analysis of specificity of anti-human leukocyte antigen antibodies in kidney recipients in reference to clinical outcome. Transplant. Proc. 46: $2606-2612$.

Pai CC, Chen M, Mirsoian A, Grossenbacher SK, et al. (2014). Treatment of chronic graft-versus-host disease with bortezomib. Blood 124: 1677-1688.

Perry DK, Pollinger HS, Burns JM, Rea D, et al. (2008). Two novel assays of alloantibody-secreting cells demonstrating resistance to desensitization with IVIG and rATG. Am. J. Transplant. 8: 133-143.

Perry DK, Burns JM, Pollinger HS, Amiot BP, et al. (2009). Proteasome inhibition causes apoptosis of normal human plasma cells preventing alloantibody production. Am. J. Transplant. 9: 201-209.

Ruangkanchanasetr P, Satirapoj B, Termmathurapoj S, Namkhanisorn K, et al. (2014). Intensive plasmapheresis and intravenous immunoglobulin for treatment of antibody-mediated rejection after kidney transplant. Exp. Clin. Transplant. 12: 328-333.

Salzedas-Netto AA, Gonzalez AM, Fagundes U, Linhares MM, et al. (2014). Financial cost of the admissions for simultaneous pancreas-kidney transplant in a Brazilian Hospital. Acta Cir. Bras. 29: 748-751.

Solez K, Colvin RB, Racusen LC, Sis B, et al. (2007). Banff '05 Meeting Report: differential diagnosis of chronic allograft injury and elimination of chronic allograft nephropathy ('CAN'). Am. J. Transplant. 7: 518-526.

Stegall MD and Gloor JM (2010). Deciphering antibody-mediated rejection: new insights into mechanisms and treatment. Curr. Opin. Organ. Transplant. 15: 8-10. 
Stegall MD, Dean PG and Gloor J (2009). Mechanisms of alloantibody production in sensitized renal allograft recipients. Am. J. Transplant. 9: 998-1005.

Sun K, Welniak LA, Panoskaltsis-Mortari A, O'Shaughnessy MJ, et al. (2004). Inhibition of acute graft-versus-host disease with retention of graft-versus-tumor effects by the proteasome inhibitor bortezomib. Proc. Natl. Acad. Sci. U S A. 101: 8120-8125

Vanderlugt CL, Rahbe SM, Elliott PJ, Dal Canto MC, et al. (2000). Treatment of established relapsing experimental autoimmune encephalomyelitis with the proteasome inhibitor PS-519. J. Autoimmun. 14: 205-211.

Wahrmann M, Haidinger M, Körmöczi GF, Weichhart T, et al. (2010). Effect of the proteasome inhibitor bortezomib on humoral immunity in two presensitized renal transplant candidates. Transplantation 89: 1385-1390.

Yang KS, Jeon H, Park Y, Jo IH, et al. (2014). Use of bortezomib as anti-humoral therapy in kidney transplantation. J. Korean Med. Sci. 29: 648-651.

Zinn MD, L'Ecuyer TJ, Fagoaga OR and Aggarwal S (2014). Bortezomib use in a pediatric cardiac transplant center. Pediatr. Transplant. 18: 469-476. 